\title{
CARACTERÍSTICAS AGRONÔMICAS DE SOJA EM FUNÇÃO DE ESPAÇAMENTOS ENTRE LINHAS DE SEMEADURA
}

\author{
Maycon Wilhann Pereira ${ }^{1}$, Leandro Meert ${ }^{2}$, Antônio Mendes de Oliveira Neto ${ }^{3}$, Naiara Guerra ${ }^{4}$, Antonio \\ Krenski ${ }^{2}$, Lucas Willwock ${ }^{2}$ \\ ${ }^{1}$ Engenheiro agrônomo. ${ }^{2}$ Centro Universitário Integrado. ${ }^{3}$ Universidade do Estado de Santa Catarina - UDESC. \\ ${ }^{4}$ Universidade Federal de Santa Catarina - UFSC. E-mail: leandro.meert@grupointegrado.br
}

\section{RESUMO}

Para a obtenção de altos rendimentos na cultura da soja é necessário que haja o mínimo de competição entre as plantas. O objetivo do trabalho foi avaliar a influência de diferentes espaçamentos sobre os componentes de rendimento e produtividade da soja. O delineamento adotado foi de blocos ao acaso com 4 tratamentos $(22,44,66$ e $88 \mathrm{~cm}$ entre linhas) e cinco repetições. A cultivar utilizada foi a 5909 com população de 204 mil plantas por hectare. As variáveis avaliadas foram altura de plantas e de inserção da primeira vagem, diâmetro do caule, número de vagens por planta, número de grãos por vagem, massa de mil grãos e produtividade. Segundo a análise de regressão, a altura de plantas e o diâmetro do caule comportaram-se de maneira quadrática, onde a medida com que um aumentou o outro reduziu. $\mathrm{O}$ maior número de vagens por planta (92) foi obtido com o espaçamento de $64 \mathrm{~cm}$ entre linhas. O número de grãos por vagens reduziu linearmente com o aumento dos espaçamentos entre as linhas. A massa de mil grãos e a produtividade apresentaram comportamento quadrático para os espaçamentos, onde a maior produtividade $4772,60 \mathrm{~kg} \mathrm{ha}^{-1}$ foi obtida com o espaçamento de $60 \mathrm{~cm}$. O diâmetro do caule apresentou correlação positiva com o número de vagens por planta e com a produtividade. A altura de inserção da primeira vagem apresentou correlação positiva com a massa de mil grãos. O número de vagens por planta foi a variável que apresentou a maior correlação com a produtividade $(0,84)$.

Palavras-chave: arranjo de plantas; competição; Glycine max; produtividade.

\section{AGRONOMIC CHARACTERISTICS OF SOYBEAN AS A FUNCTION OF SPACING BETWEEN SOWING ROWS}

\begin{abstract}
To obtain high yields in the soybean crop, it is necessary to have the minimum of competition among the plants. The objective of this work was to evaluate the influence of different spacing on productivity components and soybean yield. The design adopted was randomized blocks with 4 treatments $(22,44,66$ and $88 \mathrm{~cm}$ between rows) and six replicates. The cultivar used was 5909 with a population of 204 thousand plants per hectare. The response variables evaluated were plant height and first pod insertion, stem diameter, number of pods per plant, number of grains per pod, mass of one thousand grains and productivity. The height of plants and the diameter of the stem behaved in a quadratic manner, where the measure with which one increased the other reduced. The highest number of pods per plant (92) was obtained with the spacing of $64 \mathrm{~cm}$ between rows. The number of grains per pod reduced linearly with increasing row spacing. The mass of 1000 grains and the productivity presented a quadratic behavior for the spacing, where the highest productivity $4772.60 \mathrm{~kg} \mathrm{ha}^{-1}$ was obtained with the spacing of $60 \mathrm{~cm}$. The stem diameter showed a positive correlation with the number of pods per plant and with productivity. The height of insertion of the first pod showed a positive correlation with the mass of a thousand grains. The number of pods per plant was the variable that presented the highest correlation with productivity (0.84). Keywords: plant arrangement; competition; Glycine max; productivity.
\end{abstract}




\section{INTRODUÇÃO}

Para que a planta possa expressar o seu máximo potencial produtivo é necessário que a competição pelos recursos do meio seja minimizada, nesse contexto o principal fator que afeta a competição entre as plantas é o arranjo espacial, determinado pelo espaçamento entre as fileiras e pelo número de plantas na linha (WALKER et al., 2010).

Heiffig et al. (2006) comentaram que o máximo rendimento da soja é determinado pela maior ou menor capacidade de interceptação da radiação solar disponível e acúmulo de matéria seca tanto na fase vegetativa quanto reprodutiva, considerando que nenhum dos outros fatores são limitantes.

Sendo assim a redução no espaçamento entre as linhas de semeadura tende a reduzir o tempo para que as plantas obtenham o máximo aproveitamento possível da radiação solar incidente, além de diminuir a emergência de plantas daninhas na área, reduzindo assim a competição interespecífica (SILVA et al., 2013). Contudo isso pode criar um microclima favorável ao aparecimento de doenças (SWOBODA et al., 2011), além de poder ocorrer o auto sombreamento e a senescência precoce das folhas.

Bianchi et al. (2010) verificaram que quando o espaçamento foi reduzido de 50 para $35 \mathrm{~cm}$ entre linhas aumentou em 21\% a cobertura do solo aos 30 dias após a emergência, além de reduzir em $24 \%$ a população de plantas daninhas.

Contudo o efeito da alteração no espaçamento entre linhas sobre a produtividade ainda é controverso, alguns autores verificam resultados positivos com a diminuição do espaçamento entre linhas (AKOND et al., 2013; RAHMAN et al., 2010) e outros não (HEIFFIG et al., 2006). Isso deve-se a alta plasticidade fenotípica da cultura, a cultivar e o ambiente onde ela e cultivada (BALBINOT JUNIOR et al., 2015; BULCHING et al., 2017).

As alterações relacionadas ao espaçamento podem aumentar ou reduzir a produtividade de grãos em soja por afetar na interceptação da radiação fotossinteticamente ativa, a capacidade fotossintética do dossel e na respiração do dossel (HEIFFIG et al., 2006;
MATTIONI et al., 2008). Esses parâmetros sendo afetados podem impactar diretamente nos componentes de rendimento e por sua vez na produtividade.

Assim, se faz necessário estudos que avaliem o efeito do espaçamento entre linhas de semeadura para cultivares modernas que apresentem elevado potencial produtivo. Nesse contexto o objetivo do trabalho foi avaliar os componentes de rendimento e a produtividade da soja em função de diferentes espaçamentos entre linhas de semeadura.

\section{MATERIAL E MÉTODOS}

O trabalho foi conduzido na safra 2016/17 no município de Farol - PR, na Fazenda Planalto, situada na latitude $24{ }^{\circ} 09^{\prime} 38,44^{\prime \prime}$ S e longitude 52일 $32,14 \mathrm{O}$, com altitude de 675 metros acima do nível do mar. O solo da área é classificado como Latossolo Vermelho distroférrico de textura argilosa (EMBRAPA, 2013), as características químicas na camada de 0-20 cm apresentaram: $\mathrm{pH}=5,6\left(\mathrm{H}_{2} \mathrm{O} ; \mathrm{MO}=3,0 \%\right.$; $\mathrm{Ca}=3,4 \mathrm{cmol}_{\mathrm{c}} \mathrm{dm}^{-3} ; \mathrm{Mg}=1,6 \mathrm{cmol}_{\mathrm{c}} \mathrm{dm}^{-3} ; \mathrm{K}=0,22$ $\mathrm{cmol}_{\mathrm{c}} \mathrm{dm}^{-3} ; \mathrm{P}$ (Mehlich 1) $=12,00 \mathrm{mg} \mathrm{dm}^{-3}$ e V\%= 60. A área vem sendo manejada em sistema de semeadura direta a aproximadamente 30 anos. Antes da semeadura da soja havia trigo no local do experimento.

O delineamento experimental foi de blocos completos ao acaso com quatro tratamentos $(22,44,66$ e $88 \mathrm{~cm}$ entre linhas) e cinco repetições, cada parcela possuía $5 \times 10 \mathrm{~m}$. A cultivar utilizada foi a NS 5909 RR que possui crescimento indeterminado e grupo de maturação $5.9 \mathrm{com}$ população de 204 mil plantas $\mathrm{ha}^{-1}$, visando garantir a mesma população, após a emergência foi realizado o raleio das plantas. A semeadura foi mecanizada, realizada no dia 23 de outubro com adubação de base de $250 \mathrm{~kg} \mathrm{ha}^{-1}$ do formulado $02-20-18$, as sementes foram inoculadas com Bradyrhizobium japonicum (EMBRAPA, 2014). Ao longo da condução do trabalho o manejo fitossanitário foi realizado de acordo com as recomendações técnicas para a região. Os dados referentes a temperatura e precipitação ao longo da condução do experimento encontram-se na Figura 1. 
Figura 1. Dados de precipitação pluvial $(\mathrm{mm})$ e temperatura mínima e máxima $\left({ }^{0} \mathrm{C}\right)$ registrados no município de Farol - PR entre os meses de outubro de 2016 a fevereiro de 2017. Farol - PR, 2017.

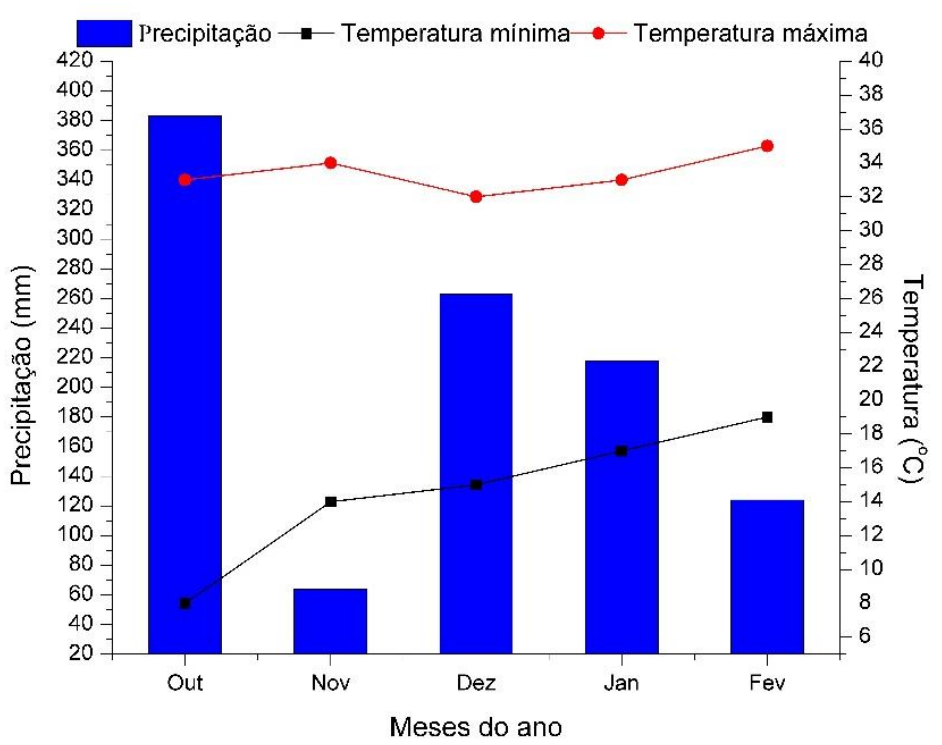

As variáveis analisadas no experimento foram altura de plantas $(\mathrm{cm})$, altura de inserção da primeira vagem $(\mathrm{cm})$ e diâmetro do caule $(\mathrm{mm})$, essas variáveis foram avaliadas quando as plantas estavam em pleno florescimento utilizando-se 10 plantas por parcela. No dia 28 de fevereiro realizou-se a colheita e em seguida avaliou-se o número de vagens por planta e número de grãos por vagem em 10 plantas por parcela. Massa de mil grãos seguindo-se a metodologia proposta por Brasil (2009) e produtividade, onde colheu-se $3 \mathrm{~m}^{2}$ de cada uma das parcelas, debulhou-se manualmente e extrapolou-se para hectare.

Após se verificar os pressupostos básicos para a análise de variância, os dados foram submetidos a análise de regressão com o auxílio do pacote estatístico Sisvar (FERREIRA, 2011).

\section{RESULTADOS E DISCUSSÃO}

Segundo a análise de regressão, a altura de plantas e o diâmetro do caule apresentaram comportamento quadrático em função da variação dos espaçamentos entre linhas. Isso ocorreu porque no menor espaçamento entre linhas $(22 \mathrm{~cm})$ houve a competição por luz entre as plantas das linhas, e a medida com que se aumentou o espaçamento a competição passou a ser entre as plantas da mesma linha. Dessa forma a menor altura de plantas foi obtida com o espaçamento de $51 \mathrm{~cm}$ (Figura 2a) e 10,4 plantas por metro. Mattioni et al. (2008) trabalhando com espaçamentos que variaram de 20 até 50 $\mathrm{cm}$, verificaram aumento linear na altura das plantas, estes autores também observaram acamamento de plantas nos maiores espaçamentos.

A competição intraespecífica por luz faz com que as plantas aloquem maior quantidade de fotoassimilados para o crescimento para que ela escape do sombreamento e não se torne uma planta dominada, com isso ocorre a redução no diâmetro do caule para as plantas com maior altura (Figura 2b). 
Figura 2. Altura de plantas $(\mathrm{cm})$ e diâmetro do caule de soja $(\mathrm{mm})$ em função de quatro espaçamentos entre linhas de semeadura. Farol - PR, 2017.

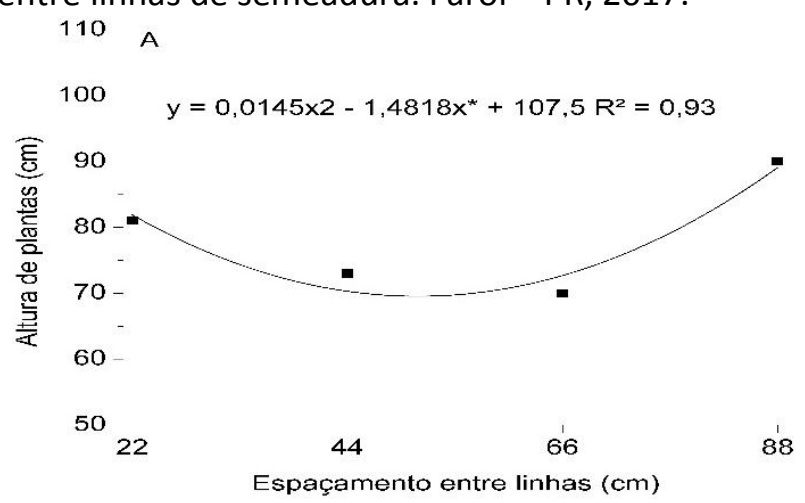

O ajuste para o número de vagens por planta de soja foi quadrático (Figura 3a), quando as plantas alcançam a melhor distribuição espacial na área o aproveitamento dos recursos do meio é máximo, e isso possibilita a elas expressarem o seu máximo potencial de produção. Contudo, a medida com que a

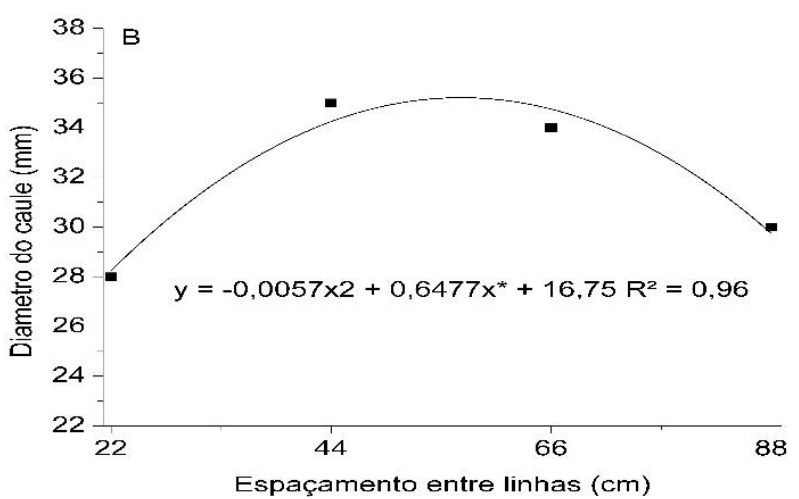

distribuição torna-se desfavorável as plantas tendem a acirrar a competição e com isso afetar negativamente os seus componentes de rendimento. No presente trabalho esta queda iniciou-se a partir de 63,9 cm entre linhas.

Figura 3. Número de vagens por planta e número de grãos por vagem de soja em função de quatro espaçamentos entre linhas de semeadura. Farol - PR, 2017.

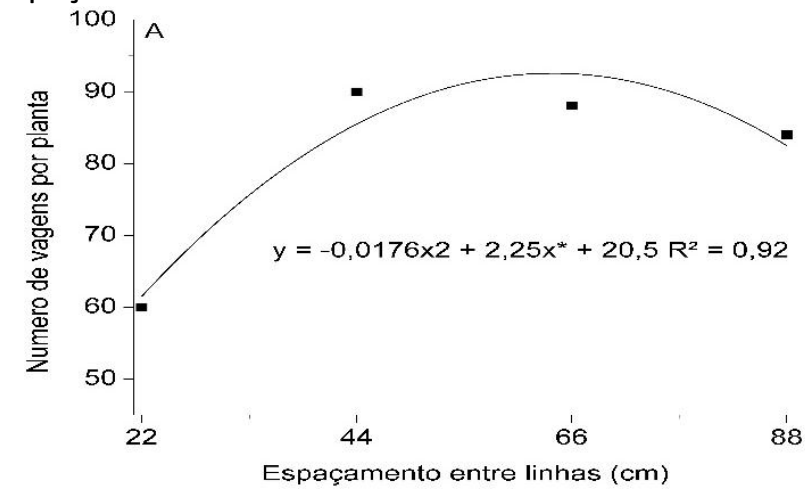

Linzmeyer Junior et al. (2008) comentam que quando aumenta-se a densidade de plantas na linha para uma mesma população, a produtividade tende a decrescer em virtude da redução no número de ramos das plantas, e o principal componente de rendimento afetado é o número de vagens por planta. A redução na quantidade dos ramos laterais é inerente a menor interceptação da luz pelas plantas.

O número de grãos por vagem, diferente dos demais componentes de rendimento, apresentou comportamento linear negativo

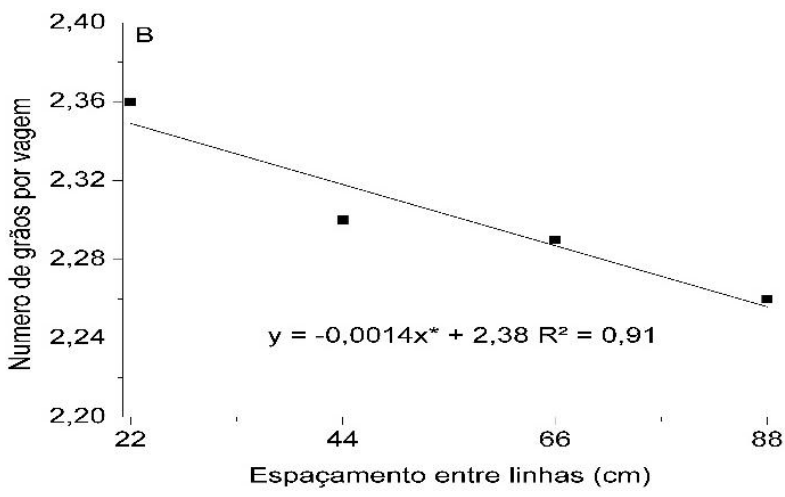

frente ao aumento nos espaçamentos (Figura 3b). Isso ocorreu porque no menor espaçamento as plantas apresentaram o menor número de vagens por planta, e isto possibilitou a formação de mais grãos por vagem, a medida com que o número de vagens aumentou, a quantidade de grãos formados reduziu, conforme pode ser verificado pela correlação linear de Pearson, onde estes dois componentes apresentaram correlação negativa $r=-0,46$ (Tabela 1 ). 
Figura 4. Massa de mil grãos (g) e produtividade de soja $\left(\mathrm{Kg} \mathrm{ha}^{-1}\right)$ em função de quatro espaçamentos entre linhas de semeadura. Farol - PR, 2017.

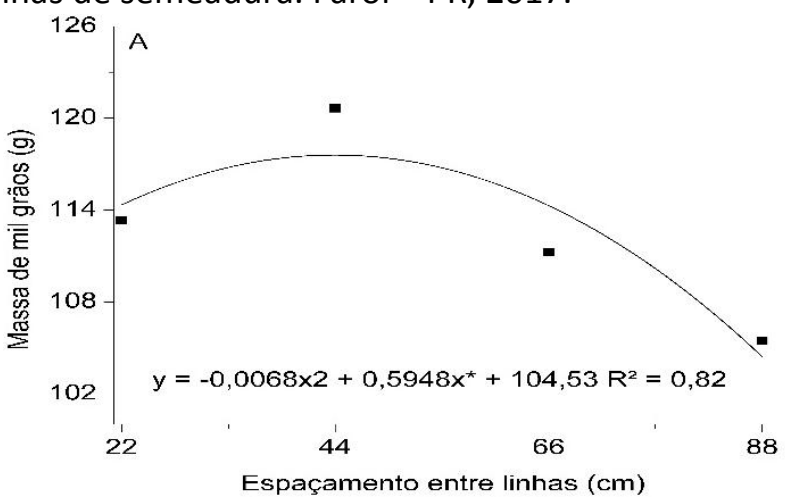

A maior massa de mil grãos foi obtida com o espaçamento de $43 \mathrm{~cm}$ entre linhas e a produtividade com o espaçamento de $60 \mathrm{~cm}$ entre linhas, a partir desses dados pode-se inferir que o espaçamento ideal para a produtividade ficou próximo ao espaçamento ideal para o número de vagens por plantas (Figura 4a e 4b).

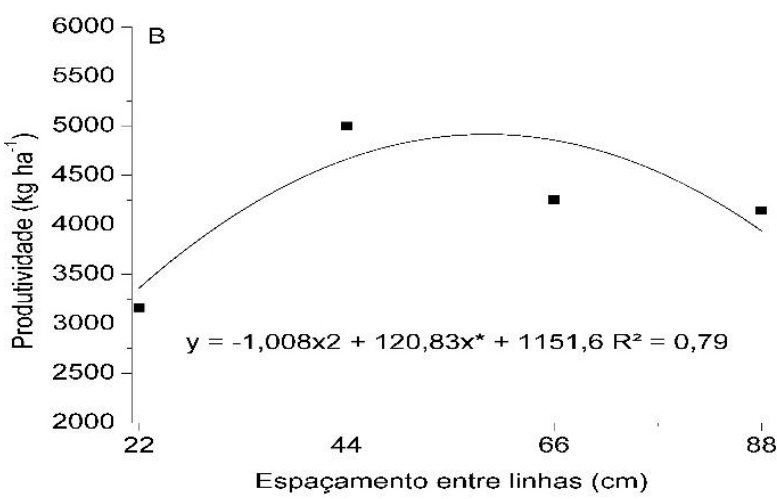

Esse resultado corrobora com Linzmeyer Junior et al. (2008) que afirmam que o principal componente de rendimento afetado pela densidade de semeadura na linha é o número de vagens por planta.

Tabela 1. Matriz de correlação linear de Pearson para altura de plantas (AP), diâmetro do caule (DC), número de vagens por planta (NVP), número de grãos por vagem (NGV), altura de inserção da primeira vagem (AIP), massa de mil grãos (MMG e produtividade (PROD) de soja em função de quatro espaçamentos entre linhas de semeadura. Farol - PR, 2017.

$\begin{array}{cccccccc} & \text { AP } & \text { DC } & \text { NVP } & \text { NGV } & \text { AIP } & \text { MMG } & \text { PROD } \\ \text { AP } & 1 & & & & & & \\ \text { DC } & -0,40 & 1 & & & & & \\ \text { NVP } & -0,27 & 0,53^{*} & 1 & & & & \\ \text { NGV } & -0,15 & -0,25 & -0,46^{*} & 1 & & & \\ \text { AIP } & 0,15 & -0,21 & -0,35 & 0,18 & 1 & & \\ \text { MMG } & -0,49^{*} & 0,30 & 0,21 & 0,22 & -0,46^{*} & 1 & 1 \\ \text { PROD } & -0,38 & 0,55^{*} & 0,84^{*} & -0,30 & -0,67^{*} & 0,60^{*} & 1\end{array}$

Significativo pelo teste de $t,{ }^{*}$ valor- $p \leq 0,05$.

De Bruin e Pedersen (2008) realizaram trabalhos no estado de lowa, EUA, entre 20042006 onde testaram o espaçamento de 28 e 76 entre linhas para a cultura da soja, os autores verificaram que no menor espaçamento a produtividade da soja foi maior nos três locais testados com elevação média de $248 \mathrm{~kg} \mathrm{ha}^{-1}$, mas o estudo mostrou que a amplitude da resposta é influenciada pelo ambiente.

Baseando-se no estande do presente trabalho, cada planta possuía teoricamente 0,048 $\mathrm{m}^{2}$ para desenvolver seu sistema radicular e também a parte área. A variação do espaçamento apenas alterou a distribuição destas plantas na área, sendo que a melhor distribuição foi proporcionada pelo espaçamento de $60 \mathrm{~cm}$ entre linhas, pois foi onde ocorreu a maior produtividade da soja $\left(4772,60 \mathrm{~kg} \mathrm{ha}^{-1}\right)$.
Estes resultados são divergentes dos verificados por Heiffig et al. (2006) que testando seis espaçamentos variando de $20-70 \mathrm{~cm}$, não encontraram efeito sobre a massa de mil grãos e produtividade. Essa divergência pode ser atribuída a cultivar utilizada no trabalho (cv Conquista) que possui tipo de crescimento determinado. Mattioni et al. (2008) observaram comportamento quadrático para a produtividade em função de diferentes espaçamentos, com o máximo rendimento obtido no espaçamento de $30 \mathrm{~cm}$.

Dentre todas as variáveis analisadas no trabalho, a que ajuda explicar melhor a produtividade obtida é o número de vagens por planta, devido a sua alta correlação com a produção de grãos $r=0,84$ (Tabela 1 ). Por outro lado, a altura de plantas e de inserção da 
primeira vagem apresentaram correlação negativa com a produtividade. Isso demonstra que quando as plantas crescem demasiadamente tendem a reduzir o número e o comprimento dos ramos laterais, diminuindo assim a superfície fotossintética e também as hastes para o surgimento de flores que posteriormente poderão se transformar em vagens (MUNDSTOCK; THOMAS, 2005; NAVARRO JÚNIOR; COSTA, 2002).

Segundo os resultados desse trabalho, o espaçamento que resultou em maior produtividade foi o de $60 \mathrm{~cm}$, espaçamento esse superior ao utilizado atualmente pela maioria dos produtores. O aumento do espaçamento atual $(45 \mathrm{~cm})$ poderia elevar o rendimento da cultura, além disso espaçamentos maiores reduzem o microclima para a ocorrência de doenças especialmente Sclerotinia sclerotiorum. Porém é necessário testar estes espaçamentos no milho, já que ambas as culturas são implantadas com o mesmo equipamento, além disso a resposta da cultura nos diferentes espaçamentos pode ser influênciada pelo ambiente da região do presente estudo.

\section{CONCLUSÕES}

Os diferentes espaçamentos entre linhas da soja afetam os componentes de rendimento e a produtividade. $O$ espaçamento de $60 \mathrm{~cm}$ entre linhas foi o que resultou em maior produtividade para a soja.

A maior correlação com a produtividade foi obtida com a variável número de vagens por planta.

\section{REFERÊNCIAS}

AKOND, M.; RAGIN, B.; BAZZELLE, R.; CLARK, W.; KANTARTZI, S. K.; MEKSEM, K.; KASSEM, M. A. Effect of Two Row Spacing on Several Agronomic Traits in Soybean. Atlas Journal of Plant Biology, v. $1, \quad$ n. 2, p. 18-23, 2013. https://doi.org/10.5147/ajpb.2013.0073

BALBINOT JUNIOR, A. A.; PROCÓPIO, S. O.; COSTA, J. M.; KOSINSKI, C. L.; PANISON, F.; DEBIASI, H.; FRANCHINI, J. C. Espaçamento reduzido e plantio cruzado associados a diferentes densidades de plantas em soja. Semina: Ciências Agrárias, v. 36, n. 5, p. 29772986, 2015. https://doi.org/10.5433/16790359.2015v36n5p2977
BIANCHI, M. A.; FLECK, N. G.; LAMEGO, F. P.; AGOSTINETTO, D. Plant arrangement and soybean cultivar roles in weed interference results. Planta Daninha, v. 28, n. 4, p. 979-991, 2010.

BRASIL. Ministério da Agricultura, Pecuária e Abastecimento. Regras para Análise de Sementes. Ministério da Agricultura, Pecuária e Abastecimento. Secretaria de Defesa Agropecuária. Brasilia: Mapa/ACS, 2009. 395p.

BULCHING, C.; OLIVEIRA NETO, A. M.; GUERRA, N.; BOTTEGA, E. L. Uso da plasticidade morfológica como estratégia para a redução de plantas em cultivares de soja. Agrarian, v.10, n.35, p.22-30, 2017. https://doi.org/10.30612/agrarian.v10i35.3931

DE BRUIN, J. L.; PEDERSEN, P. Effect of row spacing and seeding rate on soybean yield. Agronomy Journal, v. 100, n. 3, p. 704-710, 2008. https://doi.org/10.2134/agronj2007.0106

EMBRAPA. Empresa Brasileira de Pesquisa Agropecuária. Sistema Brasileiro de Classificação de Solo. 3. ed. Brasília: Embrapa, 2013. 342p.

EMBRAPA. Empresa Brasileira de Pesquisa Agropecuária. Indicações Técnicas para a Cultura da Soja no Rio Grande do Sul e em Santa Catarina, safras 2014/2015 e 2015/2016. Pelotas-RS: Embrapa, 2014. 124p.

FARIAS, E. A. P.; MOLINE, E. F. V.; SOUZA, E. F. M.; JAKELAITIS, A. Espaçamentos entre linhas $e$ épocas de aplicação de glyphosate em variedades de soja. Global Science and Technology, v. 06, n. 02, p. $24 \quad-\quad 30, \quad 2013$. https://doi.org/10.14688/1984-3801.v06n02a03

FERREIRA, D.F. Sisvar: a computer statistical analysis system. Ciência e Agrotecnologia, v.35, n.6, p.1039-1042, 2011.

https://doi.org/10.1590/S141370542011000600001

HEIFFIG, L. S.; CÂMARA, G. M. S.; MARQUES, L. A.; PEDROSO, D. B.; PIEDADE, S. M. S. Fechamento e índice de área foliar da cultura da soja em diferentes arranjos espaciais. Bragantia, v. 65, n. 2, $\quad$ 285-295, 2006. https://doi.org/10.1590/S0006 87052006000200010 
LINZMEYER JÚNIOR, R.; GUIMARÃES, F.; SANTOS D.; BENCKE, M. H. Influência de retardante vegetal e densidades de plantas sobre $\mathrm{O}$ crescimento, acamamento e produtividade da soja. Acta Scientiarum Agronomy, v. 30, n. 3, p. 373-379, 2008.

MATTIONI, F.; CORRÊA, L. A. V.; GOMES, J. C. C.; WÜNCH, J. Arranjos espaciais, plantas concorrentes e características agronômicas da soja (Glycine max (L.) merril) em cultivo orgânico. Revista Brasileira de Agrociência, v. 143, n. 4, p. 21-32, 2008.

MUNDSTOCK, C. M.; THOMAS, A. L. Soja: fatores que afetam o crescimento e o rendimento de grãos. Porto Alegre: Evangraf, 2005, 31p.

NAVARRO JÚNIOR, H. M.; COSTA, J. A. Contribuição relativa dos componentes de rendimento para a produção de grãos de soja. Pesquisa Agropecuária Brasileira, v. 37, n. 3, p. 269-274, 2002. https://doi.org/10.1590/s0100$\underline{204 \times 2002000300006}$

RAHMAN, M.; HOSSAIN, M.; ANWAR, M.; JURAIMI, A. S. Plant Density Influence on Yield and Nutritional Quality of Soybean Seed. Asian Journal of Plant Sciences, v. 10, n. 2, p. 125-132, 2011. https://doi.org/10.3923/ajps.2011.125.132

SILVA, W. B.; PETTER, F. A.; LIMA, L. B.; ANDRADE, F. R. Desenvolvimento inicial de Urochloa ruziziensis e desempenho agronômico da soja em diferentes arranjos espaciais no cerrado MatoGrossense. Bragantia, v. 72, n. 2, p. 146-153, $2013 . \quad$ https://doi.org/10.1590/S0006$\underline{87052013000200006}$

SWOBODA, C. M. P.; PEDERSEN, P.; ESKER, P. D.; MUNKVOLD, G. P. Soybean yield response to plant distribution in Fusarium virguliforme infested soils. Agronomy Journal, v. $103, \quad$ n. 6, p. 1712-1716, 2011. https://doi.org/10.2134/agronj2011.0170

WALKER, E. R.; MENGISTU, A.; BELLALOUI, N.; KOGER, C. H.; ROBERTS, R. K.; LARSON, J. A. Plant population and row-spacing effects on maturity group III soybean. Agronomy Journal, v. 102, n. 3, p. 821-826, 2010. https://doi.org/10.2134/agronj2009.0219
Recebido para publicação em 15/02/2018

Revisado em 24/08/2018

Aceito em 02/09/2018 